DOI: https://doi.org/10.46296/gt.v5i9edespmar.0056

\title{
DEPRESIÓN EN LOS ADULTOS MAYORES QUE VIVEN EN EL CENTRO GERIÁTRICO GUILLERMINA LOOR
}

\section{DEPRESSION IN OLDER ADULTS LIVING IN GULLERMINA LOOR GERIATRIC CENTER}

\author{
Sosa-Arteaga Francisco Javier ${ }^{1}$; Terán-Espinoza Juan Daniel ${ }^{2}$; \\ Sánchez-Rogel Lesly Hugo ${ }^{3}$ \\ ${ }^{1}$ Estudiante de la Maestría Académica con Trayectoria de Investigación en Psicología, Mención \\ Psicoterapia. Universidad Técnica de Manabí. Portoviejo, Ecuador. Correo: \\ fsosa9146@utm.edu.ec. ORCID ID: https://orcid.org/0000-0001-6548-8690 \\ 2 Docente de la Universidad Técnica de Manabí. Portoviejo, Ecuador. Correo: \\ juan.teran@utm.edu.ec. ORCID ID: http://orcid.org/0000-0001-6913-3360 \\ ${ }^{3}$ Estudiante de la Maestría Académica con Trayectoria de Investigación en Psicología, Mención \\ Psicoterapia. Universidad Técnica de Manabí. Portoviejo, Ecuador. Correo: \\ Isanchez1578@utm.edu.ec. ORCID ID: https://orcid.org/0000-0002-2548-9098
}

\begin{abstract}
Resumen
La depresión es una enfermedad de tipo afectivo que se presenta a menudo en las personas adultas mayores principalmente dentro de los centros geriátricos, la presente investigación se centra en conocer la realidad con base en la depresión con una muestra de 29 adultos mayores que viven en el Geriátrico Guillermina Loor de la ciudad de Portoviejo, 22 adultos mayores cumplieron con los parámetros de inclusión, la herramienta de diagnóstico fue la escala de depresión geriátrica Yesavage, esta evalúa la depresión en 3 niveles, sin depresión, probable depresión y depresión establecida, dicha escala permitió conocer la población que presenta o se encuentran desarrollando depresión y la prevalencia según el género en los adultos mayores, se mencionaron los criterios establecidos por la OMS (Organización mundial de la salud) en cuanto a la depresión, datos estadísticos proporcionados por el MIES de la realidad de los adultos mayores, los resultados fueron que el $50 \%$ no indican criterios para ser diagnosticados con este trastorno afectivo según la herramienta aplicada y el otro $50 \%$ de ellos si los presentan, teniendo una prevalencia en hombres del $60 \%$, y en mujeres $40 \%$, llegando a la conclusión de que los adultos mayores presentan depresión.
\end{abstract}

Palabras clave: Adulto Mayor, Depresión, Género, Geriátrico.

\begin{abstract}
Depression is an affective disorder that often occurs in older adults, mainly in geriatric centers, this research aims to knowledge what depression-based reality is in a sample of 29 seniors living in Guillermina Loor Geriatric Center in the city of Portoviejo, 22 of the subjects fulfilled the inclusion parameters required for this research, the diagnosis tool chosen to evaluate depression was Yesavage Geriatric Depression, this tool evaluates three levels of depression: no depression, probable depression, and established depression, such scale allowed us to determine the population with depression signs and the prevalence according to the gender in older adults, criteria about depression established by the WHO (World Health Organization) is mentioned, as well as statistics regarding seniors' reality were provided by MIES (Ministry of Economic and Social Inclusion), the results showed that $50 \%$ of the subjects did not fulfill the criteria for this affective disorder diagnosis, and the other $50 \%$ of them did present such criteria, the prevalence
\end{abstract}

Información del manuscrito:

Fecha de recepción: 06 de enero de 2022.

Fecha de aceptación: 24 de febrero de 2022.

Fecha de publicación: 04 de marzo de 2022. 
in the male population is $60 \%$, and in female population is $40 \%$, the conclusion is that older adults do experience depression.

Keywords: Older adult, Depression, Gender, Geriatric.

\section{INTRODUCCIÓN}

Según La Organización Mundial de la Salud (OMS) considera como adulta mayor a toda persona mayor de 60 años en adelante, los cambios en el individuo que llega a esta etapa no solo son físicos si no también en la salud mental donde encontramos trastornos neurocognitivos mayores, afectivos y del sueño. (Valero Pinedo, 2016), esta definición que menciona el autor puede variar debió a que depende a factores del país y las leyes que toman en consideración otros parámetros para definir a un adulto mayor.

El envejecimiento, para muchos, puede ser considerado una "época de realización, de agradable productividad y de la consolidación de habilidades y conocimientos" (Pacheco, 2016). Se debe tener en cuenta que, los recursos y funciones emocionales llegan a disminuir a consecuencia de las crisis $y$ tensiones que acumulan estas personas y que pueden provocarles problemas en su salud mental.
Según fuentes del Ministerio de Inclusión Económica y Social (MIES) en el Ecuador existen 1'254.654 adultos mayores, donde el $11 \%$ de ellos viven solos. Vale destacar que, aunque el $81 \%$ de los adultos mayores afirman estar satisfechos con su vida, el $28 \%$ se siente desamparado, por ende, vemos que la satisfacción de vida en el adulto mayor ecuatoriano crece de manera considerable cuando vive acompañado. Según perspectivas de la Organización Mundial de la Salud (OMS), entre los años 2000 y 2050 el porcentaje de adultos mayores se duplicará: de $11 \%$ al $22 \%$, es decir, que pasará de 605 millones a 2.000 millones de habitantes de esta población del número total expuesto por parte del MIES el $14,9 \%$ fue o es víctima de abandono y parte de esta población se encuentra en los centros geriátricos que los acogen (Diario El Telégrafo, 2017).

Respecto a la presencia de adultos mayores, en el país, es el MIES el 
encargado de brindar atención especializada a las personas de la tercer a edad que corresponden a la población de 65 años y más, esta actividad la ejecuta a través de 14 centros geriátricos públicos, los que llegan a sostenerse con aportes y convenios que el Gobierno hace con organizaciones públicas y privadas (Diario El Telégrafo, 2017).

El adulto mayor llega a experimentar varios cambios psicológicos de carácter emocional como el temor, hostilidad, ansiedad, inseguridad, desasosiego o depresión. Pero es el temor al abandono los que lleva a que caigan en un sentimiento afligido y preocupante a diario (Pontificia Universidad Católica de Chile, 2016).

La Organización Mundial de la Salud señala a la depresión como un trastorno caracterizado por la manifestación de pérdida de placer o interés, tristeza, trastornos del sueño o del apetito, sentimientos de baja autoestima o de culpa, falta de concentración y sensación de cansancio. Ésta puede llegar a hacerse recurrente 0 crónica $y$ obstaculizar el desempeño en la escuela o el ambiente de trabajo y la capacidad para enfrentar la vida diaria (Loje Cantinnet, 2018).

La Clasificación Internacional de Enfermedades (CIE-10), la conceptualiza como los episodios típicos, tanto leves como moderados - graves, el paciente sufre un decaimiento del ánimo, con reducción de su energía y disminución de su actividad. Se deterioran la capacidad de disfrutar, el interés y la concentración, y es frecuente un cansancio importante, incluso después de la realización de esfuerzos mínimos. Habitualmente el sueño se halla perturbado, en tanto que disminuye el apetito. Casi siempre decaen la autoestima y la confianza en sí mismo, y a menudo aparecen algunas ideas de culpa o de ser inútil, incluso en las formas leves. El episodio depresivo puede ser calificado como leve, moderado - grave, según la cantidad y la gravedad de sus síntomas (OPS, 2008).

La reacción de un adulto mayor ante el abandono como sostiene Marín (2003, pág. 56); sostiene que al observar que junto a él no existe la presencia de alguien que le brinde el apoyo necesario en sus actividades, 
le toca asimilar en primer lugar que tiene que seguir adelante en todo lo que haga de una manera solitaria, y esa situación que induce a una decisión no la toma como algo positivo, ya que esto lo que puede provocar, entre varias sintomatologías, la tristeza que en algunos casos puede ir más allá y convertirse en un cuadro de más gravedad, como la depresión, siendo este un problema de magnitud que afectan de manera enorme e inmediata su actual situación ya sea dentro 0 fuera de un centro geriátrico.

Entonces se puede decir que el adulto mayor en estos centros vive una realidad donde su etapa de envejecimiento se marca por la marginación que le expresa la sociedad en su conjunto, lo que provoca pensamientos $y$ sentimientos negativos a sí mismo, como el de sentir que no aporta en nada tanto en su familia y mucho menos hacia la sociedad como parte productiva de la misma. Y es ahí donde aparece el sufrimiento al experimentar y asumir aquellas dolencias de su edad, como el enfrentar la lejanía, el abandono e incluso el irrespeto y desamor del entorno que lo rodea.

(Calderon M, 2018) menciona con respecto a la depresión en relación con el adulto mayor que influyen varios factores, tanto genéticos, biológicos, psicológicos y ambientales. Varios factores anatómicos tratan de explicar la depresión, entre ellos el volumen diferente de hipocampo, parahipocampo, amígdala y corteza prefrontal. La etiología vascular parece asociarse con la depresión tardía.

La OMS reporta una cifra ponderada de depresión del 10,4\%. La prevalencia de los síntomas depresivos en los pacientes adultos varía entre el 8 y $16 \%$ en los diferentes estudios. La prevalencia de depresión mayor varía entre el 1 y 4\%. La depresión mayor varía ampliamente en rangos $2,3 \%$ a $25 \%$ en países como España, Estados Unidos y Australia. En la India los episodios depresivos fueron de $12,7 \%$. De manera global la depresión en Estados Unidos fue de $11,1 \%$. En México la prevalencia fue de 21,7 \% (Fernández Arce \& González Reyes, 2016). 
Según (Da Silva Rodrígues, Carvalho Figueiredo, \& Ramos Frausto, 2018) destaca que la depresión en el adulto mayor no solo afecta el estado de ánimo, debido a que repercute en otras áreas, estudios han verificado que la depresión geriátrica presenta un incremento de más del $50 \%$ en los TNC (trastornos neuro cognitivos) cuando es evaluada en muestras clínicas, y casi un $40 \%$ en muestras comunitarias. Estas cifras son significativas dado que la depresión afecta el funcionamiento óptimo neuropsicológico de los adultos mayores, potenciando las pérdidas de memoria y éstas, a su vez, pueden estar asociadas con algunas dificultades de orientación, de aprendizaje y de reconocimiento, entre otros aspectos.

La realización de esta investigación se basa en conocer si existe depresión o un índice de trastorno depresivo en el adulto mayor dentro del geriátrico Guillermina Loor de la ciudad de Portoviejo, esto permitirá describir la realidad emocional que se viven los adultos mayores dentro de dicho centro geriátrico.
En este sentido es fundamental determinar los principales padecimientos relacionados con el estado mental y emocional del adulto mayor que reside en los centros geriátricos, analizar el estado actual en el que viven los adultos mayores en aquellas instituciones, de los adultos mayores en situación de abandono en los centros geriátricos; $y$ con base a esto elaborar estrategias que contribuyan a mejorar el estado mental y emocional del adulto mayor, a través de un accionar oportuno de tipo preventivo en pro del bienestar de estas personas.

Las personas en todas sus etapas de vida, perciben la vejez desde una perspectiva negativa; se expresa el temor de llegar a la vejez y se niegan a aceptar que es un proceso natural del ser humano como parte del ciclo vital, de cierto modo se puede evidenciar que la vejez ha sido vista desde muchos ángulos, y formas humanas de expresión, que diariamente las personas asocian esta etapa de vida con enfermedad, la muerte, minusvalía adquiriendo de este modo actitudes, creencias y acciones que fomentan la predisposición, el rechazo a esta 
etapa de vida propia del ser humano. (Luengo, Araneda, \& López, 2010)

(Viguera, 2010) manifiesta que cuando la persona adulta mayor ha dejado de ser independiente, la familia se constituye en su único soporte, sin embargo, en muchos casos el hogar se convierte en un espacio de marginación, abandono y de maltrato. En los últimos años constatamos que la familia ha sufrido múltiples cambios, según este autor sostiene que estos cambios a lo largo de los tiempos afectan el desarrollo por la razón que los miembros de la familia pierden el interés por mantener una calidad de vida para el adulto mayor $y$ vulnerando sus derechos.

Es importante tener en consideración los números cambios que las personas experimentan cuando llegan a la adultez mayor no están limitados solo a los físicos y psicológicos también se suman los sociales y encomios como lo afirman (Bentosela \& Mustaca, 2005) la vejez acarrea un sin fin de transformaciones que se hacen presentes no solo a nivel físico sino también a nivel psicológico, social y económico. Este autor menciona como estos componentes forman parte del proceso y las afectaciones como el abandono genera problemas en estos cambios propios de la adultez mayor.

Un estudio realizado (Roldan Mozón, 2007) muestra como el abandono puede generar afectaciones en el adulto mayor basados en especialistas en geriatría indican que es frecuente observar actualmente situaciones en las cuales la familia cercana manifiesta cansancio, agotamiento, desinterés, agresión. El adulto mayor se vuelve más dependiente, fácil de influenciar por el temor a ser abandonado, se abandona a sí mismo, se deprime, e incluso, algunas veces se vuelve, por el contrario, hostil y agresivo, este estudio muestra como el abandono genera una condición de peligro para el adulto mayor que no está recibiendo los cuidados necesarios por parte de sus familiares.

La depresión en un adulto mayor tiene un impacto que va a llegar a trascender en sus vidas, causada por dos factores básicos, primero por la situación en que se encuentran y por su característica psicofisiológica que conlleva ser un ser humano en dicha etapa de la vida. En el adulto mayor la depresión va a generar una 
influencia muy notable en el comportamiento, en sus relaciones, en sus interacciones, en sus conductas y en sus comportamientos, y en su esfera afectiva.

Para poder realizar esta investigación se estableció como objetivo general lo siguiente:

- Identificar depresión en los adultos mayores que viven en el centro geriátrico Guillermina Loor de la ciudad de Portoviejo.

- Así mismo también hemos determinado 3 objetivos específico que ayudaron delimitar la investigación:

- Establecer el nivel de depresión que manifiestan los adultos mayores dentro del geriátrico.

- Reconocer la prevalencia de género ante la depresión en el adulto mayor.

- Relacionar la depresión con el abandono en los adultos mayores del centro geriátrico Guillermina Loor.

\section{METODOLOGÍA}

El presente trabajo investigativo es de tipo descriptivo, transversal y con un enfoque cuantitativo y cualitativo, de manera que se puedan abarcar aspectos generales y específicos sobre el tema planteado. Además de que lleven a conocer parte de la vida de los participantes de la investigación, así como su propio mundo, relacionados con el fenómeno en estudio. La importancia de este tipo de estudio recae en la posibilidad de obtener descripciones de las experiencias sobre el fenómeno en estudio, tal y como las viven los sujetos.

La investigación se realizó en el centro geriátrico Guillermina Loor de la ciudad de Portoviejo, que se encuentra ubicado dentro de la provincia de Manabí.

La investigación se realizó en un lapso de 6 meses durante el 20202021 datos proporcionados por la institución geriátrica Guillermina Loor.

La población estuvo conformada por 29 adultos mayores de ambos sexos (hombres y mujeres), que estén registrados en el centro geriátrico, y que cumplan con los criterios de inclusión para la siguiente investigación.

Criterios de Inclusión: 
- Adultos mayores con edades comprendidas entre los 65 y 85 años.

- Adultos mayores en uso de sus facultades cognitivas

- Adultos mayores que deseen participar voluntariamente $\mathrm{y} / \mathrm{o}$ que les sea autorizado por sus cuidadores y/o la autoridad de la institución.

Criterios de exclusión:

- Adultos mayores con demencia senil.

- Adultos mayores con discapacidad auditiva.

- Adultos mayores que se nieguen a participar de manera voluntaria para la investigación.

- Adultos mayores que no se encuentren presentes en los días de la recolección de la información.

La herramienta empleada fue la escala geriátrica de Yesavage, este instrumento se encuentra estandarizado para la identificación de la depresión, consta de quince reactivos con dos opciones a elegir, se determinó este método para evaluar la depresión en los adultos mayores, es importante mencionar que es un instrumento utilizado a nivel gerontológico, con niveles aceptables de confiabilidad, dentro de distintas prácticas. (Butista Valdivia, 2016)

La forma de calificación de la escala geriátrica se presenta de la siguiente manera, solo se maneja por asignación los puntajes de 1 y 0 , por lo que las respuestas que indican depresión se les asigna el puntaje de 1 y a las que indican lo contrario se les asigna un 0, para esclarecer la forma de calificación se procede a mencionar que las preguntas que son respondidas con un "SI" son las que presentan probabilidad de una presente depresión y son calificadas con un 1 con excepción de las siguientes respuestas, en este caso son las preguntas número $1,5,7,9$, 16, 19, 21, 27, 29, 30. (Butista Valdivia, 2016)

Es importante mencionar que las personas que tienen un puntaje de 0 al 10 son consideradas sin depresión, las que tienen un resultado de 11 al 30 se consideran personas con depresión. (Butista Valdivia, 2016)

La escala fue previamente aplicada por los psicólogos de la institución y con los resultados obtenidos se va realizar la investigación descriptiva, los resultados se empleó el 
programa de IBM SPSS Statistics, el autor declara que no hay conflicto de interés y confidencialidad en la información.

La muestra a investigada son 22 adultos mayores que corresponde a una edad de 65 años en adelante, se excluyeron adultos mayores que presentaron enfermedades de tipo psiquiátricas, aquellos que manifestaron un deterioro cognitivo significativo.

Declaración de los aspectos éticos: Oficio dirigido al geriátrico para la obtención de dichos datos, datos procesados con SPSS, no hay conflicto de interés $y$ confidencialidad de la información.

Tabla №1. Escala de depresión geriátrica Yesavage aplicada a los adultos mayores del geriátrico Guillermina Loor

\begin{tabular}{rrrrr}
\hline & Frecuencia & Porcentaje & $\begin{array}{c}\text { Porcentaje } \\
\text { válido }\end{array}$ & $\begin{array}{c}\text { Porcentaje } \\
\text { acumulado }\end{array}$ \\
\hline No depresión & 11 & 50,0 & 50,0 & 50,0 \\
$\begin{array}{c}\text { Probable } \\
\text { depresión }\end{array}$ & 8 & 36,4 & 36,4 & 86,4 \\
$\begin{array}{c}\text { Depresión } \\
\text { establecida }\end{array}$ & 3 & 13,6 & 13,6 & 100,0 \\
Total & 22 & 100,0 & 100,0 & \\
\hline
\end{tabular}

Fuente: Elaboración Propia.

Se obtuvo como resultado de 22 una probable depresión y un 13,6\% adultos mayores evaluados que la en una depresión establecida existe prevalencia de la depresión es de un un $50 \%$ que no presenta depresión. $50 \%$ distribuidos con un $36,6 \%$ en

Tabla №2. Se especifica la relación de la depresión con la condición del género en el adulto mayor.

Escala de depresión geriátrica Yesavage

\begin{tabular}{crrr} 
& \multicolumn{2}{c}{ Género del adulto mayor } & Total \\
\cline { 2 - 3 } & Masculino & Femenino & \\
\cline { 2 - 3 } Depresión establecida & 2 & 1 & 3 \\
Probable depresión & 4 & 4 & 8 \\
No depresión & 6 & 5 & 11
\end{tabular}


Fuente: Elaboración Propia.

Los resultados de la Tabla 2, mostró que de 11 adultos mayores que presentaron probable depresión y depresión establecida la prevalencia es de $60 \%$ (6 hombres) en hombres a diferencia de las mujeres que obtuvieron el $40 \%$ (5 mujeres) de predisposición a desarrollar depresión.

\section{DISCUSIÓN}

En la presente investigación descriptiva se obtuvo como resultado que la prevalecían de la depresión en los adultos mayores del centro geriátrico Guillermina Loor es de un $50 \%$ distribuidos con un $36,6 \%$ en una probable depresión y un 13,6\% en una depresión establecida existe un $50 \%$ que no presenta depresión, esto guarda relación con un estudio realizado por el doctor Marco Vinicio Sinchire Gómez en los centros geriátricos de la ciudad de Loja obtuvo que a 130 adultos que acuden a los centros geriátricos de Malacatos y Vilcabamba un $36 \%$ de adultos mayores posee un grado de depresión y 64\% no padece depresión (Sinchire Gómez, 2016), estos datos nos dan a comprender que existe un margen diferencial $14 \%$ entre estos estudios pero así mismo nos rectifican la presencia de trastornos depresivos en los centros geriátricos.

Sin embargo, estos resultados mantiene una relación con la investigación realizada en la casa hogar Beato Enrique Rebuschin del distrito Surquillo en Perú obtuvo que el " $55 \%$ de los adultos mayores corresponde al nivel "Depresión moderada"; seguido del $43 \%$ que está "Sin depresión"; por último, el $3 \%$ posee un nivel "Depresión severa" (Bachiller Giannina Rodríguez, 2019), estos datos coinciden con resultados obtenidos en el geriátrico Guillermina Loor.

Dentro de los resultados obtenidos en el centro geriátrico Guillermina Loor dio a conocer que los hombres representan un $60 \%$ (6 hombres) a diferencia de las mujeres que obtuvieron el $40 \%$ (5 mujeres ) de predisposición a desarrollar trastornos de tipo afectivo, esto discrepa de la investigación realizada por la universidad de 
Cartagena en el año 2016 que donde se observó que un " $48,7 \%$ de los adultos mayores fueron mujeres y estas eran más propensas a deprimirse". (Fernandez Arce \& González Reyes, 2016)

En Ecuador se han realizado pocos estudios, la mayor parte de ellos en adultos mayores hospitalizados 0 residentes de asilos. Un estudio en pacientes residentes en asilo encontró depresión en $70 \%$ (Calderon M, 2018), esto mantiene una correlación el trabajo de investigación realizado dentro del geriátrico Guillermina Loor dando constancia que la depresión dentro de estos lugares de acogida está (Butista Valdivia, 2016) presente y que en muchas ocasiones son tomadas como desapercibidas dentro de esta última etapa de vida donde debe primar el bienestar no solo físico si no también psicológico.

\section{CONCLUSIONES}

- Una vez obtenidos los resultados se puede indicar que dentro del geriátrico Guillermina Loor existen trastornos afectivos latentes y la mitad de los adultos mayores que residen dentro de la institución mantiene un predominio a desarrollar una depresión establecida.

- Se pudo constatar que un $50 \%$ de los adultos mayores no presentan ningún tipo de trastornos afectivos, esto puede generar la hipótesis que poseen mecanismo de afrontamiento psicológicos ante la depresión.

- Existe un índice mayoritario dentro de este geriátrico en donde los adultos mayores hombres tiene prevalencia a diferencia de las mujeres para poder desarrollar depresión.

- Con base a todo el dato obtenido se concluye que se debe emplear un plan psicoterapéutico direccionado a la prevención y promoción de la depresión en los adultos mayores así también terapias focalizadas al grupo que ya presentan este tipo de trastorno y así mismo a los que mantiene una probabilidad de poseerlo.

\section{BIBLIOGRAFÍA}

Bachiller Giannina Rodríguez, R. Q. (2019). La depresión en pacientes adultos mayores oncológicos de la casa hogar 
Beato Enrique Rebuschini en el distrito de Surquillo. UNIVERSIDAD INCA GARCILASO DE LA VEGA, 51. Obtenido de: http://repositorio.uigv.edu.pe/ bitstream/handle/20.500.1181 8/5003/TRSUFICIENCIA_RO DR\%c3\%8dGUEZ\%20QUISP E.pdf?sequence $=1$ \&isAllowed $=\mathrm{y}$

Bentosela, M., \& Mustaca, A. (2005). Efectos cognitivos $y$ emocionales del envejecimiento: aportes de investigaciones basicas para las estrategias de rehabilitación. Obtenido de: https://www.redalyc.org/pdf/1 80/18022205.pdf

Butista Valdivia, J. (2016). Depresión en el adulto mayor por abandono familiar. Revista Académica de Investigación, 248. Obtenido de: https://www.eumed.net/rev/tla temoani/22/depresion.pdf

Calderón M, D. (2018). Epidemiología de la depresión en el adulto mayor. Med Hered, 184. Obtenido de: http://www.scielo.org.pe/pdf/r $\mathrm{mh} / \mathrm{v} 29 \mathrm{n} 3 / \mathrm{a} 09 \mathrm{v} 29 \mathrm{n} 3 . \mathrm{pdf}$

Da Silva Rodrigues, C. Y., Carvalho Figueiredo, P., \& Ramos Frausto, V. M. (2018). La depresión y su influencia en los cambios neuropsicológicos del adulto mayor con trastorno neurocognitivo leve debido a la enfermedad de Alzheimer.
CES Psico, 71. Obtenido de: https://revistas.ces.edu.co/ind ex.php/psicologia/article/view/ $4417 / 2953$

Diario El Telégrafo. (15 de Junio de 2017). El abandono afecta al $14 \%$ de adultos mayores. Diario El Telégrafo. Obtenido de

https://www.eltelegrafo.com.e c/noticias/41/4/el-abandonoafecta-al-14-de-adultosmayores

Fernandez Arce, M., Guzmán Gárcia, L., Torres, N., Romero Gárcia, K., \& González Reyes, D. (2016). PREVALENCIA DE DEPRESIÓN EN EL ADULTO MAYOR EN LOS CENTROS DE VIDA EN CARTAGENA. Cartagena: Universidad de Cartagena. Obtenido de: https://repositorio.unicartagen a.edu.co/bitstream/handle/11 227/3098/PREVALENCIA\%2 ODE\%20DEPRESION\%20EN \%20LOS\%20CENTROS\%20 DE\%20VIDA\%20DE\%20CAR TAGENA\%20INFORME\%20 FINAL.pdf?sequence=1\&isAll owed $=\mathrm{y}$

Loje Cantinnet, R. (2018). Depresión $Y$ calidad de vida en usuarios del centro del adulto mayor de la red asistencial ANCASH, Chimbote. UDALECH, 1. Obtenido de: http://repositorio.uladech.edu. pe/bitstream/handle/1234567 89/5970/DEPRESION_CALID AD_DE_VIDA_LOJE_CANTI NETT_RICARDO_ALCIDES. 


\section{pdf? sequence $=1$ \&isAllowed $=$ $y$}

Luengo, C., Araneda, G., \& López, M. (2010). Factores del cuidador familiar que influyen en el cumplimiento de los cuidados básicos del usuario postrado. Scielo, 19(1). Obtenido de: https://scielo.isciii.es/scielo.ph p?script=sci_arttext\&pid=S11 32-12962010000100003

Marín, J. (2003). Envejecimiento (Vol. III). Madrid, España: Sociedad Española de Geriatría y Gerontología, Salud Pública Educ. Obtenido de

http://webs.uvigo.es/mpsp/rev 03-1/envejecimiento-03-1.pdf

OPS. (2008). Clasificación Estadística Internacional de enfermedades y problemas relacionados con la salud. Washington: International Statistical Classification of Diseases and Related Health Problems. Obtenido de: http://ais.paho.org/classificati ons/chapters/pdf/volume1.pdf

Pacheco, M. (2016). Repercusión del abandono en el estado mental y emocional del adulto mayor que reside en el asilo Ancianos Hogar San José. Loja: Universidad Nacional de Loja. Obtenido de https://dspace.unl.edu.ec/jspu i/bitstream/123456789/9878/1 /TESIS\%20MANUEL\%20ANI
BAL\%20PACHECO\%20SAN CHEZ.pdf

Pontificia Universidad Católica de Chile. (2016). Proceso de envejecimiento: sus implicancias biológicas y sociales. Obtenido de http://escuela.med.puc.cl/pagi nas/udas/Geriatria/Geriatria

Roldan Mozón, A. V. (2007). Vivencias del adulto mayor frente al abandono de sus familiares en la comunidad Hermanitas descalzas. Universidad Nacional Mayor de San Marcos, 12. Obtenido de:

https://core.ac.uk/download/p df/323352179.pdf

Sinchire Gómez, M. V. (2016). Factores que influyen en la depresión en los adultos. Loja: Universidad nacional de Loja facultad de medicina. Obtenido de: https://dspace.unl.edu.ec/jspu i/bitstream/123456789/19621/ 1/Factores\%20que\%20influye n\%20en\%20la\%20depresi\%c $3 \%$ b3n\%20en\%20los\%20adu Itos $\% 20$ mayores $\% 20$ que $\% 20$ acuden\%20a\%20los\%20cent ros\%20gerontol\%c3\%b3gicos $\% 20 d e . p d f$

Valero Pinedo, L. (2016). Salud y calidad de vida del adulto mayor. Rev Perú Med Exp Salud Publica, 199-200. Obtenido de: http://www.scielo.org.pe/pdf/ri ns/v33n2/a01v33n2.pdf 